\title{
ERS Lung Science Conference 2016 report
}

\author{
Bertrand De Meulder ${ }^{1}$, Bruno Crestani ${ }^{2,3}$ and Ian M. Adcock ${ }^{4}$
}

\begin{abstract}
Affiliations: 'European Institute for Systems Biology and Medicine, CNRS-ENS-UCBL, Université de Lyon, Lyon, France. ${ }^{2}$ APHP, Hôpital Bichat, Service de Pneumologie A, Centre de Compétences des Maladies Pulmonaires Rares, Paris, France. ${ }^{3}$ Université Paris Diderot, Sorbonne Paris Cité, INSERM Unité 1152, Paris, France. ${ }^{4}$ Airway Disease Section, National Heart and Lung Institute, Imperial College London, London, UK.
\end{abstract}

Correspondence: Ian M. Adcock, Airway Disease Section, National Heart and Lung Institute, Imperial College London, Dovehouse Street, London, SW3 6LY, UK. E-mail: ian.adcock@imperial.ac.uk

0 @ERSpublications

The 14th ERS LSC took place on March 10-13, 2016, with the theme of "system approaches in lung disease" http://ow.ly/4ncU5W

The aims of the European Respiratory Society (ERS) Lung Science Conference (LSC) are three-fold: to present the very best of international lung science research; to highlight new discoveries likely to impact on the future of respiratory medicine; and to encourage debate and interaction between young post-doctoral scientists and established investigators. The 14th ERS LSC took place on March 10-13, 2016, with the theme of "system approaches in lung disease" and included a number of talks relating to why systems medicine will be increasingly important in defining new phenotypes of patients in lung diseases, particularly in relation to personalised medicine and in elucidating novel drug targets. Other talks highlighted the advances in 'omic technology that has enabled the concept of personalised 'omics to occur, and we also saw the future in how modelling of systems may bring new understanding of disease mechanisms and how linking social media to artificial intelligence models may direct patient-orientated treatment. These featured talks were supported by a large number of posters and abstract talks highlighting the impressive research being undertaken by young researchers in this area, and more generally in cell and molecular biology of lung disease.

The research presented crossed the boundaries between respiratory disease and other disciplines, including mathematics and bioinformatics, with the aim to enhance investigators' own research programmes and to provide innovative therapies to better treat patients. Some patients with chronic lung diseases have long been recognised as difficult to treat due to their complexity, and the presence of subtypes of disease and even different diseases masquerading as the same disease. Indeed, as described by Peter Sterk (University of Amsterdam, Amsterdam, the Netherlands), asthma in general and particularly severe asthma is a heterogeneous spectrum, demonstrating multiple and varying biological pathways to be associated with the disease in individual patients [1]. This has fuelled the search of subphenotypes of (severe) asthma with the ultimate goal of providing so-called endotypes [2].

In an attempt to understand this spectrum of disease, a large number of different 'omics samples were obtained from the airways, blood and urine of $>600$ adults subjects in the Unbiased Biomarkers in Prediction of Respiratory Disease Outcomes (U-BIOPRED) consortium with the aim to capture molecular networks. For example, severe asthmatics are characterised by multiple, significantly different proteomic clusters in peripheral blood showing that eosinophilia in severe asthma comprises significantly different molecular phenotypes. Bertrand De Meulder demonstrated the value of having standard protocols for obtaining these types of data, and the types of analysis tools that can be used to analyse and integrate these data sets, and thereby create the first comprehensive "handprints" that identified distinct sputum and blood asthma handprints. Personalised medicine will increasingly rely on such systems medicine approaches to define and refine patient subphenotypes. For all analysis of 'omics data, several generic steps

Received: April 252016 | Accepted after revision: April 272016

Conflict of interest: Disclosures can be found alongside this article at err.ersjournals.com

Provenance: Submitted article, peer reviewed.

Copyright CERS 2016. ERR articles are open access and distributed under the terms of the Creative Commons Attribution Non-Commercial Licence 4.0. 
must be observed to provide meaningful data. These include data management and cleaning, quality control, feature filtering, 'omics-based clustering and biomarker identification. Finally, the results must be validated, ideally in an independent cohort and with experimental data [3,4]. Pre- and peri-natal exposure to cigarette smoking acts through epigenetic mechanisms. Using the population-based German birth cohort LISAplus and the mother-child cohort LINA, and disease models, Irina Lehmann (Helmholtz Centre for Environmental Research, Leipzig, Germany) demonstrated persistent differential DNA methylation patterns in enhancer regions of genes in subjects whose mothers smoked and linked these to impaired lung function in early childhood [5].

Similar approaches have been undertaken in other lung diseases. Rosa Faner (Fundació Clínic per a la Recerca Biomèdica, Barcelona, Spain) reported on the progress made regarding chronic obstructive pulmonary disease (COPD) associated with smoking, the incidence of which increases in the ageing population. Utilising a systems biology and network analysis approach it was possible to identify disease modules and relevant pathogenic pathways such as those relating to B-cells in subsets of patients [6]. These provide mechanistic understanding of COPD subtypes and new concepts for the treatment of clinical traits [7]. The incidence of idiopathic pulmonary fibrosis (IPF) also increases with age, and IPF is a progressive and devastating chronic lung disease, characterised by altered cellular composition and accumulation of extracellular matrix with a worse prognosis than that of lung cancer. Oliver Eickelberg (Comprehensive Pneumology Center (CPC), University Hospital, Ludwig-Maximilians-University and Helmholtz Zentrum München, Munich, Germany) described how analysing large datasets of transcriptomic and proteomic data from disease cells in an unbiased fashion contributed greatly to the identification of novel cellular and molecular mechanisms and signalling pathways indicating the existence of distinct phenotypes [8]. Soni Savai Pullamsetti (Dept of Lung Development and Remodelling, Max Planck Institute for Heart and Lung Research, Bad Nauheim, Germany) described how epigenetics, including DNA methylation, modifications in chromatin structure and changes in noncoding RNA expression, are important in determining how environmental and lifestyle factors (diet, hypoxia and drug/toxins) affect the susceptibility to pulmonary hypertension. Importantly, pulmonary hypertension vascular cells maintain a "stable" and "heritable" phenotype in cell culture, enabling the dissection of the regulatory events that drive disease, and further details are provided an the accompanying article in this issue of the European Respiratory Review (ERR) [9].

The advances in this approach to disease subphenotyping have relied on the improvements in 'omic platforms. Assessment of the extent of cellular heterogeneity, for example, transitional stages of differentiation and dynamic changes in gene expression within individual cells, has only recently been possible with the advent of single-cell RNA sequencing (RNA-seq) (Yan $\mathrm{Xu}$, Divisions of Pulmonary Biology, Perinatal Institute and Biomedical Informatics, Cincinnati Children's Hospital Medical Center, Cincinnati, OH, USA). Yan $\mathrm{Xu}$ also highlighted the success of the LungMAP consortium and the development of a computational pipeline for Single Cell RNAseq Profiting Analysis (SINCERA), which enables analysis using standard desktop/laptop computers [10]. Metabolomics is the global unbiased analysis of the small-molecule metabolites within a biological system, under a given set of conditions. A metabolomics profile incorporates the preceding steps in the 'omics cascade (e.g. proteome, transcriptome and genome) to provide an integrative phenotype (Craig Wheelock, Dept of Medical Biochemistry and Biophysics, Division of Physiological Chemistry II, and Centre for Allergy Research, Karolinska Institutet, Stockholm, Sweden). Initial analysis of urinary metabolites in U-BIOPRED has defined distinct molecular subphenotypes of asthma and similar patent stratification is seen with COPD in the Karolinska COSMIC (COPD and Seretide: a Multi-Center Intervention and Characterization) COPD cohort. One advantage of metabolomics is that it enables the analysis of both endogenous metabolites and those originating from exogenous sources (microbiome, diet, therapeutics, etc.) [4, 11].

The power of integrating all these approaches together was exemplified by Rui Chen (Dept of Genetics, Stanford University School of Medicine, Stanford, CA, USA) who described how an iPOP (integrative Personal Omics Profile) analysis approach in a single "healthy" adult can reflect changes in physiological states over time [12]. Thus, it was possible to determine "novel biomarkers" for the onset of type 2 diabetes and those that reflect respiratory viral infections. This approach provides a comprehensive view of the biological pathways that change during disease onset and progression, and will, in turn, lead to the identification of molecular signatures of disease and personalised health monitoring, early diagnostics and precision medicine.

A critical aspect of systems biology is the construction of mathematical models to reflect the disease or system that can then be used to make predictions of drug response, for example. The power of this type of analysis was highlighted by Jason Bates (Dept of Medicine, University of Vermont College of Medicine, Burlington, VT, USA) using models of lung physiology to advance our knowledge of asthma and acute lung injury [13]. Using a model of metabolic and signalling networks involved in cellular responses to hypoxia, Lindsay Edwards (Centre of Human and Aerospace Physiological Sciences, School of Biomedical 
Sciences, King's College, London, UK) demonstrated that it was possible to demonstrate a gap in basic knowledge in tricarboxylic acid cycle biology that resulted in the discovery of mutations in these genes among Tibetans that enabled survival at high altitude [14]. It must be recognised that no single model is always correct and models must be developed for the question being asked.

The environment affects a subject's subphenotype and measurement of "the totality of human environmental exposures from conception onwards, complementing the genome" [15] represents an aspect of epidemiology known as the exposome (Valerie Siroux, INSERM U823, Equipe Épidémiologie Respiratoire, Grenoble, France). The exposome approach is complex, both in term of comprehensively measuring it (hundreds of exposures need to be considered) over the life course and of analysing its relation to health. Despite this, it is likely that a better understanding of environmental factors will lead to improved prevention strategies in asthma [16]. Jørgen Vestbo (Centre for Respiratory Medicine and Allergy, The University of Manchester and University Hospital South Manchester NHS Foundation Trust, Manchester, UK) described three different epidemiological approaches to address scientific questions that cannot be tested in a laboratory, clinical or clinical trial setting. Using a prospective cohort study design and merging data from three cohorts to study the trajectories of lung function decline over 25 years, it was possible to document that maximally attained lung function in early adulthood (small lungs) is probably as important as excess decline in forced expiratory volume in $1 \mathrm{~s}$ for the development of COPD. In addition, the beneficial effect of statins on COPD exacerbations was observed using a large nested case-control design [16]. Further details of these approaches are given in accompanying articles in this issue of the ERR $[16,17]$.

The recent rapid development of high-throughput technologies linked to mobile technologies has given rise to a new type of epidemiology (Marcel Salathé, Center for Infectious Disease Dynamics, Dept of Biology, Penn State University, University Park, PA, USA). Digital data sources can provide local and timely information about disease and health dynamics in populations around the world, and importantly, a large percentage of the online population publicly shares information on social media. Indeed, digital disease surveillance augmented traditional approaches in the 2013 outbreak of influenza A (H7N9). In combination with the growth of electronic data records from traditional health systems, new paradigms, combining patient-generated data from search engines, internet discussion boards, social media platforms, mobile apps and others with data from traditional health systems, have emerged using their complementary strengths, particularly in relation to infectious disease surveillance and adverse drug event monitoring [18].

In the future, mobile and social media data from phones could indicate a change in the health/disease status and/or exposure to potentially detrimental environmental challenges specific to an individual subject. This may then be interpreted by one of the artificial intelligence programmes being developed, such as Dr Watson (Pascal Sempé, IBM Watson Health, Bois-Colombes, France). This presents an unprecedented opportunity to transform the ways in which we manage our health, and the advantages of cloud-based analysis and interpretation of health risk, and align this with the reimbursement models of payers. This is dependent upon the success of these programmes in delivering quality assessments and good clinical outcomes following disease diagnosis. Today, it is possible for the IBM Watson Health Cloud to turn everyday routines into health data that can be shared to help doctors provide better and more personalised car,e taking advantage of clinical, research and social data from a diverse range of health sources in real time.

A key aspect of the LSC is the presentations by young investigators. The standard overall was exceptionally high and selection of prize winners was difficult. The William MacNee award was presented to Eva Maria Garrido-Martin (University of Southampton, Southampton, UK) for her work on transcriptomic profiling of macrophages isolated from human non-small cell lung carcinoma (NSCLC) to reveal novel macrophage subsets using various bioinformatic and cell biology techniques. Tumour-associated macrophages (TAMs) were isolated from 45 resected NSCLC specimens and macrophages were isolated from adjacent healthy tissue by fluorescence-activated cell sorting, and high-throughput RNA-seq was performed on these samples. This revealed that the genetic profile of TAMs is dramatically reprogrammed and exhibits a mixed M1/M2 phenotype. In addition, TAMs from squamous cell carcinoma have unique features compared to adenocarcinoma. High infiltration of immune cells within the tumour, which is associated with good prognosis, may be determined by specific properties of the macrophages, highlighting their importance in driving an optimum immune response.

Aina Martin (CPC, Munich, Germany) was awarded the Best Oral Presentation prize for her work on how extracellular vesicles modulate noncanonical Wnt signalling in pulmonary fibrosis. A comprehensive profiling of extracellular vesicles from bronchoalveolar lavage fluid was undertaken and she discovered that Wnt5A was strongly upregulated in vesicles from fibrosis patients. Wnt5A potentiated pro-fibrotic transforming growth factor- $\beta$ signalling in lung epithelial cells and thus represents a potential target for new therapies in IPF. All other short oral presenters and poster awardees are to be congratulated on their work, and encouraged to support the ERS by submitting their work for the International Congress. 
Next year, the LSC (March 23-26, 2017) will focus on common mechanisms in chronic lung injury and lung cancer. The new conference and seminars director, Rachel Chambers (University College London, London, UK), has already established an exciting programme with excellent speakers. We encourage you to submit abstracts, and plan to join the LSC community and build the future of European respiratory science.

\section{References}

Sterk PJ. Chronic diseases like asthma and COPD: do they truly exist? Eur Respir J 2016; 47: 359-361.

Shaw DE, Sousa AR, Fowler SJ, et al. Clinical and inflammatory characteristics of the European U-BIOPRED adult severe asthma cohort. Eur Respir J 2015; 46: 1308-1321.

3 Saqi M, Pellet J, Roznovat I, et al. Systems medicine: the future of medical genomics, healthcare, and wellness Methods Mol Biol 2016; 1386: 43-60.

4 Wheelock CE, Goss VM, Balgoma D, et al. Application of 'omics technologies to biomarker discovery in inflammatory lung diseases. Eur Respir J 2013; 42: 802-825.

5 Bauer $\mathrm{T}$, Trump $\mathrm{S}$, Ishaque $\mathrm{N}$, et al. Environment-induced epigenetic reprogramming in genomic regulatory elements in smoking mothers and their children. Mol Syst Biol 2016; 12: 861.

6 Faner R, Cruz T, Casserras T, et al. Network analysis of lung transcriptomics reveals a distinct B cell signature in emphysema. Am J Respir Crit Care Med 2016 [in press DOI: 10.1164/rccm.201507-1311OC].

7 Agusti A, Bel E, Thomas $\mathrm{M}$, et al. Treatable traits: toward precision medicine of chronic airway diseases. Eur Respir J 2016; 47: 410-419.

8 Meiners S, Eickelberg O, Konigshoff M. Hallmarks of the ageing lung. Eur Respir J 2015; 45: 807-827.

9 Chelludurai P, Werner Seeger W, Pullamsetti SS. Epigenetic mechanisms in pulmonary arterial hypertension: need for global perspectives. Eur Respir Rev 2016; 25: 135-140.

10 Du Y, Guo M, Whitsett JA, et al. "LungGENS": a web-based tool for mapping single-cell gene expression in the developing lung. Thorax 2015; 70: 1092-1094.

11 Wheelock AM, Wheelock CE. Trials and tribulations of 'omics data analysis: assessing quality of SIMCA-based multivariate models using examples from pulmonary medicine. Mol Biosyst 2013; 9: 2589-2596.

12 Chen R, Snyder M. Promise of personalized omics to precision medicine. Wiley Interdiscip Rev Syst Biol Med 2013; 5: 73-82.

13 Bates JH, Smith BJ, Allen GB. Computational models of ventilator induced lung injury and surfactant dysfunction. Drug Discov Today Dis Models 2015; 15: 17-22.

14 Edwards LM, Thiele I. Applying systems biology methods to the study of human physiology in extreme environments. Extrem Physiol Med 2013; 2: 8.

15 Wild CP. Complementing the genome with an "exposome": the outstanding challenge of environmental exposure measurement in molecular epidemiology. Cancer Epidemiol Biomarkers Prev 2005; 14: 1847-1850.

16 Siroux V, Agier L, Slama R. The exposome concept: a challenge and a potential driver for environmental health research. Eur Respir Rev 2016; 25: 124-129.

17 Vestbo J. Natural experiments and large databases in respiratory and cardiovascular disease. Eur Respir Rev 2016; 25: $130-134$.

18 Salathé M, Bengtsson L, Bodnar TJ, et al. Digital epidemiology. PLoS Comput Biol 2012; 8: e1002616. 